\title{
EDITORIAL
}

DOI: http://dx.doi.org/10.19053/22160159.5215

\section{TECNOLOGÍAS DE LA INFORMACIÓN Y LA COMUNICACIÓN APLICADAS A LA EDUCACIÓN}

La sociedad de la información y el conocimiento ha proporcionado uno de los soportes más sólidos — pregonado por Manuel Castells, y otros analistas-, considerando que las Tecnologías de la Información y la Comunicación (TIC), han cobrado un papel protagónico en el siglo XXI, donde se han dado nuevas relaciones que se transforman, y emergen otras alternativas para tener acceso a la educación. Cada vez se multiplican más los programas académicos relacionados con las modalidades: presencial con apoyo de TIC, b-learning o aprendizaje mixto con apoyo de las (TIC), o el aprendizaje e-learning, aprendizaje en línea. Estas han sido formas en las cuales se han considerado otros ambientes de aprendizaje en escenarios diferentes, entendiendo que el conocimiento no sólo se adquiere en el aula de clase, permitiendo pensar que la creación de nuevos escenarios con elementos innovadores ha generado y transformado el conocimiento y hace ver que no sólo es el conocimiento científico el que lo genera. Así lo consideró Drucker (citado por Rojas, 2006): no es una inspiración, la acción de innovar "requiere esfuerzo sistemático y alto grado de organización.” (p. 30)

En lo relacionado con la información, como lo cita Micheli (2002), Drucker afirma:

Lo que llamamos revolución de la información es de hecho una revolución del conocimiento, es la reorganización del trabajo tradicional, basado en siglos de experiencia, mediante la aplicación del conocimiento y en especial del análisis sistemático y lógico. La clave no es la electrónica sino la ciencia cognitiva. Eso significa que para mantener el liderazgo en la economía y en la tecnología, influye la posición social que tengan los profesionales del conocimiento y en la aceptación social de sus valores. Lo que implica para ellos ser considerados "empleados" tradicionales sería equivalente al trato que en Inglaterra (durante la revolución industrial) se dio a los tecnólogos, como si fueran comerciantes. (p. 524) 
Las TIC han sido parte de un movimiento social sin precedentes en la historia de la humanidad, y han generado un impacto en la sociedad, lo cual se visibiliza desde hace quinientos años, al utilizar el libro como soporte impreso. Al hablar de las TIC se tiene en cuenta que son una oportunidad y un desafío, donde se encuentra un sentido, como es el de permitir desarrollar sociedades más democráticas e inclusivas, que puedan articularse con la colaboración, la creatividad y la distribución tal vez más justa del conocimiento científico, con una educación equitativa y de calidad para todos. Las TIC deben mirarse como el conjunto de tecnologías que permiten el acceso, producción, tratamiento y comunicación de información presentada en diferentes formatos y a la vez entrelazada con el texto, la imagen y el sonido.

Cabero (1998) afirma que las TIC

son las que giran en torno a tres medios básicos: la informática, la microelectrónica y las telecomunicaciones; pero giran, no sólo de forma aislada sino, lo que es más significativo, de manera interactiva e interconexionadas, lo que permite conseguir nuevas realidades comunicativas. (p. 198)

En el contexto iberoamericano se ha visto la necesidad de generar políticas que ayuden a dar directrices y lineamientos claros sobre la apropiación y uso de las tecnologías; para el caso de Colombia, a partir del año 2007, el gobierno Nacional ha propuesto políticas para el uso y apropiación de las TIC, y es así como el 30 de julio de 2009, el Ministerio de TIC (MINTIC), promulga la Ley 1341 por la cual se definen principios y conceptos sobre la sociedad de la información y la organización de las Tecnologías de la Información y las Comunicaciones. Posterior a esta ley, el Ministerio de Educación Nacional (MEN) y el MINTIC, generan alianzas con el fin de proponer la creación de Centros de Innovación Educativa Regional, denominados los CIER; de esta manera el Proyecto

CIER del Ministerio de Educación Nacional promoverá la construcción de capacidades regionales de uso educativo de las Tecnologías de la Información y la Comunicación (TIC), para mejorar la calidad de las prácticas educativas en las instituciones y entidades del sistema educativo colombiano y para aportar a la reducción de la brecha educativa entre las regiones del país. ${ }^{1}$

1 Tomado de: http://www.colombiaaprende.edu.co/html/micrositios/1752/w3-propertyname-3020.html. Mayo 15 de 2016. 
La alianza entre diferentes entidades como COLCIENCIAS, MEN y MINTIC, ha permitido generar algunos proyectos para propiciar la incorporación de las TIC, en los contextos educativos, y ha venido evolucionando en los últimos años; esto ha sido una fortaleza y un apoyo para el sistema educativo, afectando a los actores en el proceso de aprendizaje y asegurando de alguna manera una mejora en la calidad de la educación en las diferentes modalidades. Ha logrado promover aspectos como la apropiación y uso de las TIC, donde se genera innovación tecnológica, el fortalecimiento de la producción de conocimiento pedagógico y la apropiación social del conocimiento; así lo ha considerado uno de los proyectos desarrollados, en conjunto con varias entidades, por la Representación Ontológica Hipermedial. Una de las publicaciones del libro Representación Ontológica hipermedial en línea para el aprendizaje significativo (Londoño, 2015), ha evidenciado estos tres aspectos importantes, en respuesta a la necesidad de hacer productivo el conocimiento con la implementación de las TIC, con el fin de construir representaciones ontológicas, cuando se incorpora en el desarrollo de las prácticas educativas de cursos en línea b-learning o presenciales en diversas áreas del saber, de igual forma en el desarrollo y evaluación de proyectos educativos hipermediales y colaborativos.

Las TIC, han evolucionado a un ritmo vertiginoso, con una responsabilidad social por parte de la Instituciones de Educación Superior (IES); se ha visto la necesidad de incorporarlas en la educación, lo cual se ha realizado y se visibiliza en las investigaciones adelantadas al respecto, generando procesos de reflexión desde el punto de vista de las diferentes modalidades y niveles educativos en cada uno de los programas de formación profesional, involucrando a los actores en los nuevos escenarios y ambientes de aprendizaje, al igual que la incorporación de las políticas derivadas de la Integración de las TIC.

En las IES, las TIC se han convertido en un eje transversal en todos los planes curriculares en cualquiera en las áreas del conocimiento - por mencionar las Artes, la Medicina, la Música entre otras-, considerándolas como eje fundamental generador de competencias digitales para profesores y estudiantes, apropiadas y pertinentes en cada una de las disciplinas; la aplicabilidad que tienen las TIC ha sido uno de los compromisos adquiridos por las IES en la formación de profesionales en la diversidad de áreas del conocimiento, así como de investigadores dedicados al desarrollo de la Ciencia y la Tecnología, con investigación desde el aula de clase hasta investigación científica, pasando por la social, en donde cada uno de los 
resultados posiblemente responde a unas necesidades, y genera un impacto en cada una de las personas involucradas en la aplicabilidad de las TIC.

De esta manera se evidencia y visibiliza cómo las TIC logran irradiar en cada uno de los niveles de escolaridad, con una motivación para los profesores y estudiantes, al lograr involucrar las TIC en su quehacer diario, y así se ha logrado verificar una vez más en los resultados de investigaciones como se ve en el proyecto "La Producción del conocimiento experiencial de los estudiantes en la Educación Superior". Este proyecto hace referencia a una investigación desarrollada en colaboración con la Universidad Central "Marta Abreu" de Las Villas (UCLV) en Cuba, y la Universidad Libre de Bruselas (VUB) en Bélgica, durante los años 2010 a 2014, con el auspicio del Consejo Interuniversitario Flamenco (VLIR) que tuvo como culminación la defensa de una tesis doctoral en Ciencias de la Educación. El proyecto de investigación asociado a esta tesis doctoral fue financiado por VLIR-UOS (programa de colaboración interuniversitaria con la Universidad Central "Marta Abreu" de Las Villas). En este artículo de reflexión se expone una síntesis de la metodología utilizada para el desarrollo del proyecto de investigación y sus conclusiones. Se considera también que en los niveles de educación Básica y Media se han desarrollado investigaciones que tienen que ver con las TIC, donde se muestra cómo las políticas nacionales del MEN se articulan en los diferentes niveles de escolaridad.

En cuanto al proyecto de investigación relacionado con las "Competencias TIC para el desarrollo profesional docente en Educación Básica”, da a conocer los resultados de una investigación en instituciones educativas de la ciudad de Cúcuta sobre competencias TIC y su integración a la práctica pedagógica. El propósito consistió en identificar y relacionar los niveles de competencias TIC, que reportan los docentes de Básica, considerando su perfil de formación y tomando como referencia el modelo propuesto por el Ministerio de Educación Nacional (MEN) de Colombia en el año 2013. Se trata de una investigación cuantitativa de enfoque descriptivo correlacional en la cual se miden los niveles de las competencias TIC del modelo MEN a partir de las dimensiones de competencias presentes en los docentes. Se seleccionaron 255 docentes de 16 instituciones educativas, que respondieron un instrumento de escala tipo Likert. De manera global, los resultados muestran que los docentes exhiben un buen nivel de competencias TIC. Las competencias auto reportadas en las distintas dimensiones, por otra parte, muestran correlaciones altas y significativas entre sí, lo cual demuestra que se 
trata de un conocimiento consolidado y que existe una integración parcial de las TIC en el aula.

Determinar que las TIC no sólo entran a jugar un papel importante en el proceso educativo, sino examinar que realmente están presentes y se requieren en los procesos de gestión, facilitando de cierta forma el manejo eficiente y eficaz de la información es tema analizado en la parte administrativo-académica.

Entre las consideraciones que tienen las TIC se halla el uso y aplicabilidad en cada uno de los procesos, y ver la transformación que pueden generar como herramientas mediadoras en los procesos de aprendizaje y no sólo como la instrumentalización. Uno de los artículos señala algunas transformaciones vivenciadas en la era de la información, en cuyo abordaje metodológico de enfoque fenomenológico, se analiza y describe para luego discutir no sólo como aparatos técnicos y su dimensión instrumental, sino también examinar sus interrelaciones con el poder y con la reproducción del sistema capitalista. En la primera parte, la relación de conceptos como sociedad, tecnología y cultura evidencia que la vida se sustenta en el inmediatismo, la inmersión y la rapidez del acceso en un mundo tecnológico, trayendo consigo múltiples fracturas que aquí son repensadas desde la perspectiva docente. En un segundo plano, entra la discusión temática de las tecnologías en su contexto a saber, partiendo del modelo fordista hacia las condiciones de trabajo docente relacionando la enseñanza superior y la innovación tecnológica y, en la última parte, expone cómo pensar y lidiar con la tecnología aprovechando sus posibilidades pedagógicas, culturales y políticas. Como conclusión, se enfatiza el potencial educativo y finalidades del proceso tecnológico hoy.

Poder vivenciar cada una de las experiencias cuando se incorporan las TIC en los procesos de aprendizaje, en el aula de clase y fuera de ella son tecnologías que hoy en día se requieren no como un artículo de lujo, sino como una herramienta que potencializa la educación y que se requiere a diario; es algo que vivimos desde el manejo de competencias digitales de docentes de Básica Primaria de una institución educativa, a través de actividades con tabletas, consideradas como una de las tecnologías móviles a fin de consolidar una cultura digital. La incorporación de dispositivos digitales individuales para estudiantes y docentes de los distintos niveles educativos, se está transformando en una tendencia mayoritaria en las actuales políticas gubernamentales de inclusión digital, inclusión social y de mejoramiento de las oportunidades educativas de los sistemas educativos de Iberoamérica. 
Otro de los artículos hace referencia a lograr que las TIC tengan la facilidad de incurrir en los diferentes saberes y áreas del conocimiento; es algo trascendental y relevante como lo muestra el artículo que menciona las "Relaciones entre Logro de Aprendizaje, Automonitoreo, Estilo Cognitivo y Estilos de Aprendizaje en Estudiantes de Medicina”, con el análisis de las relaciones que existen entre logro de aprendizaje, uso de auto-monitoreo metacognitivo, estilo cognitivo (dimensión Dependencia-Independencia de Campo) y estilo de aprendizaje (Clasificación de Grasha), en estudiantes universitarios. El estudio se realizó con una población de 130 alumnos de una Facultad de Medicina de Sexto a Décimo semestres en una universidad en Bogotá, Colombia. Utilizando la metodología de correlaciones bi-variadas se buscaron asociaciones entre el promedio académico alcanzado por los estudiantes, el automonitoreo metacognitivo, medido con el cuestionario MSQL, los estilos cognitivos por medio de la prueba EFT y los estilos de aprendizaje reportados en el inventario de Grasha. Los resultados mostraron correlaciones positivas del logro de aprendizaje con las variables: estilo cognitivo, uso de estrategias metacognitivas, estilos de aprendizaje competitivo y participativo, y correlaciones negativas con los estilos de aprendizaje evasivo y dependiente. De igual forma se encontraron otras correlaciones entre las variables de estudio que son discutidas en el documento.

Considerando que las TIC están articuladas de forma transversal en las diferentes áreas del conocimiento, como se describe desde el arte el cual ha sido importante y donde han tenido origen algunas de las TIC: por mencionar la virtualidad que nace desde la magia, considerada la Reina de las Artes, desde lo escénico como se evidencia en un artículo que describe algunas de las emociones del espectador en una experiencia fotográfica, pictórica y cinematográfica, donde se exponen los resultados del análisis comparativo de tres estudios: The science of art: $A$ neurological theory of aesthetic experience (1999), Neural correlates of beauty (2004) y Neurocinematics: The neuroscience of film (2008). Cada una de las investigaciones señaladas examina la actividad neuronal de un grupo de participantes que observa fotografías, pinturas o filmes. Se consideró que las experiencias expuestas permiten comprender el alcance y objeto del neurocinema. El método de la investigación fue revisión documental y el enfoque analítico. Se diseñaron tablas para sistematizar la revisión de los textos y se elaboraron tablas descriptivas basadas en resúmenes analíticos RAE. Se concluye que dos de los estudios tuvieron en común que los participantes presentaron actividad neuronal en el área del sistema límbico, la región del cerebro que procesa las emociones. De la forma en que cada una de las acciones realizadas está 
monitoreada por nuestro sistema neuronal —en la vida y sobre todo en la actividad educativa - , para que el proceso tenga validez debe ser monitoreado a través de un proceso de razonamiento y evaluación, lo que nos garantiza que realmente hubo un proceso de aprendizaje, que se valida con la praxis, confrontada por la teoría. Es lo que se da en el artículo que tiene que ver con la "Evaluación de Competencias con apoyo de un Sistema de Gestión de Aprendizaje", el cual presenta los resultados de una investigación descriptiva correlacional con dos propósitos definidos: por un lado, analizar la opinión de los estudiantes respecto a la contribución de la metodología de evaluación de los proyectos formativos para su proceso de formación por competencias; por otro lado, hacer un análisis comparativo entre los resultados de la evaluación de evidencias realizada por los estudiantes y la evaluación del profesor. La experimentación se realizó con estudiantes de un curso de lógica formal de pregrado del programa de Ingeniería de Sistemas y Computación de la Universidad del Quindío, Colombia. Para soportar el proceso de evaluación de competencias se desarrolló un nuevo componente tecnológico que se incorpora como un bloque al sistema de gestión de aprendizaje Moodle. Los resultados del trabajo permiten afirmar que los estudiantes consideran que la participación activa en el proceso de evaluación contribuye al desarrollo de las competencias del curso. Con relación a los resultados de la evaluación de evidencias, se identificó que existe diferencia estadísticamente significativa entre la evaluación realizada por los estudiantes y la evaluación del profesor.

Pero como en el proceso educativo se debe tener en cuenta el razonamiento dentro de los debates propiciados, se busca mirar si uno o más juicios son válidos o existe la falsedad, y es lo que el autor logra destacar en la investigación que abordó desde "El razonamiento como eje transversal en la construcción del pensamiento lógico", dando a conocer las formas de razonamiento intuitivo que emplearon los estudiantes del Grado Quinto de la Institución Educativa Rural del Sur en el aula de clase. Para identificar estas formas se desarrollaron tres etapas: inicialmente se realizó un reconocimiento de la población; en segundo lugar, se observaron y se grabaron algunas clases de asignaturas como Español, Inglés y Matemáticas, en las cuales se identificaron las principales formas de lenguaje cotidiano empleadas por los estudiantes en el aula: atribución de sentido e interacción. Por último, partiendo de los planteamientos de la teoría fundamentada, se analizó la información recaudada en el software Atlas.ti con el fin de identificar las categorías emergentes e inductivas presentes entre los datos, y sus respectivas relaciones, que pusieron de manifiesto las formas de razonamiento; se logró establecer que los estudiantes que participaron en la investigación usaron 
principalmente la paráfrasis y aposición como una forma de razonamiento cotidiano; además se observó que la experiencia fue un factor de gran influencia en la interpretación de situaciones del entorno.

Es así como este número de la Revista Praxis \& Saber logra evidenciar lo más relevante de las TIC, desde su uso, su aplicabilidad, hasta la contrastación con la evaluación y el razonamiento de las mismas en la vida académica, contemplando la gestión en los procesos educativos y como eje articulador de competencias válidas en la vida profesional de cualquier sujeto social.

Olga Nájar Sánchez

Editora Invitada

\section{Referencias}

Cabero, A. Julio (1998). Las nuevas tecnologías de la información y la comunicación: aportaciones a la enseñanza. en: Cabero, J. (Ed.): Nuevas Tecnologías aplicadas a la educación. Madrid: Síntesis, pp. 15-38.

Drucker, P.F. (1994). La Sociedad postcapitalista. Bogotá: Norma, p. 207. Londoño, O. L. (2015) Representación Ontológica hipermedial en línea para el aprendizaje significativo, bajo la Dirección Editorial de Olga Lucía Londoño Palacio. Bogotá, pág. 14.

Micheli, T, Jordi. (2002). Digitofactura: Flexibilización, internet y trabajadores del conocimiento. México: Revista Comercio Exterior, junio de 2002. Vol. 52, No 6. pág. 522-536.

Rojas, Q, J. M. (2006) Gestión educativa en la sociedad del conocimiento. Bogotá: Cooperativa Editorial Magisterio. 